\title{
Prematuridad y estimulación temprana: ¿un binomio determinante para la prevención de la discapacidad?
}

\section{Linda María Madriz Bermúdez** Lorena Mora Hernández***}

\author{
*Hospital de Especialidades I.S.S.S.T.E.Py Estancia Infantil \#2 del mismo Instituto en Puebla, México; jorgebax@hotmail.com \\ ** Cátedra de la Conceptualización de la Educación Especial de la Universidad Estatal a Distancia en San José, Costa Rica; \\ Imadriz@uned.ac.cr. \\ *** Cátedra de la Conceptualización de la Educación Especial de la Universidad Estatal a Distancia en San José, Costa Rica; \\ Imorahernandez@yahoo.com
}

Recibido: 6 de setiembre de 2011

Aceptado: 14 de noviembre de 2011

\begin{abstract}
RESUMEN
Diferentes estudios han señalado que el nacimiento prematuro puede tener secuelas sobre el desarrollo del niño o la niña. En la actualidad, investigadores, médicos, enfermerasypedagogos, venenlaestimulacióntemprana una herramienta para la prevención y tratamiento de las consecuencias de un nacimiento anticipado. El presente artículo revisa y estudia una selección de investigaciones que han abordado la discusión de temas relacionados con la prematuridad, la estimulación temprana y la prevención de la discapacidad, para integrar y analizar sus resultados con el objetivo de dar sustento a una nueva investigación, interesada en formular un protocolo de atención temprana para la prevención de la discapacidad en niños y niñas nacidos antes de tiempo y con factores de riesgo biológico asociados. En este sentido, una revisión sistemática de las publicaciones relacionadas con estos temas constituye el primer paso para alcanzar esta meta que ha sido claramente definida, para el beneficio de la población infantil participante en este estudio, avalado por la Vicerrectoría de Investigación de la Universidad Estatal a Distancia y de otras que consideren su pertinencia. Vale la pena recordar que una revisión sistemática es la síntesis formal, cualitativa, basada en un protocolo minucioso, que incluye investigaciones de calidad metodológica comparable que tienen en común la misma intención de sintetizar la evidencia científica. Bajo esta dirección, la exposición que se efectúa respeta el protocolo de construcción que define una revisión sistemática para que los lectores se familiaricen con cada uno de los pasos que llevan implícitos y que pueden
\end{abstract}

\begin{abstract}
SUMMARY
Different studies have shown that premature birth may have consequences on the development of the child. Currently, researchers, doctors, nurses and teachers, use early stimulation as a tool for prevention and treatment of the consequences of an early birth. This article reviews and studies a range of investigations that have addressed the discussion of issues related to prematurity, early stimulation and prevention of disability to integrate and analyze their results with the objective of sustaining a new research interest in formulating an early treatment protocol for the prevention of disability in children born prematurely and associated biological risk factors. In this sense, a systematic review of publications related to these issues is the first step towards this goal, which has been clearly defined, for the benefit of children participating in this study, endorsed by the Vice-Rector of Research at Universidad Estatal a Distancia (UNED) and others they deem relevant. It is worth remembering that a systematic review is the formal synthesis, qualitative, based on a detailed protocol, including research of comparable methodological quality which share the same intention of synthesizing the scientific evidence. Under this direction, the exposure is made, respecting the construction protocol that defines a systematic review for readers to become familiar with each of the steps leading embedded and have been taken into account within the theoretical development process research that interests.
\end{abstract}


ser tomados en consideración dentro del proceso de elaboración del marco teórico de investigaciones futuras.

\section{PALABRAS CLAVE}

Atención, estimulación e intervención temprana; prevención; discapacidad; prematuridad; condición de riesgo.

\section{KEY WORDS}

Attention, stimulation and early intervention, prevention, disability, prematurity, a condition of risk.

\section{INTRODUCCIÓN}

Este artículo versa sobre la revisión sistemática de varias investigaciones recientes relacionadas con el tema en discusión sobre prematuridad y estimulación temprana como estrategias para la prevención de la discapacidad.

Se analizaron algunos artículos que se vinculaban con estos tópicos en Costa Rica y México, con el fin de reconocer las acciones inmediatas que se han venido desarrollando cuando se da el nacimiento prematuro de un niño o niña y que, en alguna medida, han procurado mitigar el impacto de una eventual discapacidad asociada a esta condición.

\section{METODOLOGÍA}

Este trabajo investigativo realiza un estudio exploratorio descriptivo a partir del cual se pretende conocer las acciones que, desde el ámbito hospitalario público en dos centros médicos de dos países diferentes, se siguen para orientar a padres de familia o encargados en la búsqueda de una estimulación y atención temprana, cuando se da el nacimiento de un niño en condiciones de prematuridad y con factores de riesgo biológico. De igual forma, pretende explorar la atención que se brinda en el ámbito educativo al incorporarse la estimulación temprana a servicios de guardería, cuando la opción de padres de familia o encargados es ingresarlos a ellos por su situación laboral o por la necesidad de cuido responsable.

La investigación se desarrolla bajo un enfoque mixto por tratarse de un proceso que recolecta, analiza y vincula datos cuantitativos y cualitativos en un mismo estudio para responder al planteamiento de un problema. En el caso de la investigación que nos ocupa, se retomará el impacto de este enfoque por considerar la complementariedad de la información cualitativa con la cuantitativa, al brindar una percepción de lo que ninguno de los dos podría conseguir por separado. Así, se pretende diagnosticar y analizar la atención integral que reciben los niños nacidos con factores de riesgo biológico asociados a prematuridad para, a partir de los datos obtenidos y la información que de ellos se desprenda, poder conocer las necesidades de apoyo para esta población desde el enfoque de Atención Temprana. El insumo obtenido pretenderá demostrar entonces la relevancia de la creación de un Programa de Atención en esta área que responda a las necesidades de la población.

Este artículo surge dentro del marco de una revisión sistemática de diferentes estudios en Costa Rica y México, que se realiza como parte de la investigación para buscar respuestas a la siguiente pregunta:

¿Cuál es el abordaje que se ha dado a la relación entre prematuridad y estimulación temprana en Costa Rica y México?

Para responder a esta interrogante, se seleccionaron trabajos finales de graduación para optar por grados académicos de licenciatura y maestría en distintas disciplinas dentro de 
la Universidad de Costa Rica. Además, fueron consultados artículos científicos de distintas revistas costarricenses y mexicanas en formato digital.

Los documentos incluidos en esta revisión fueron seleccionados a partir de los siguientes criterios:
a. Temas relacionados con discapacidad, prematuridad y estimulación temprana.
b. Actualidad: los documentos en estudio se generaron del año 1990 al año 2010.

\section{c. Origen:}

»En el caso de Costa Rica, se dio prioridad al análisis de textos creados por estudiantes de instituciones académicas reconocidas, como por ejemplo la Universidad de Costa Rica, para optar por un grado de licenciatura o maestría.

\begin{abstract}
»Los artículos científicos, tanto costarricenses como mexicanos, fueron extraídos de revistas y bases de datos reconocidas e indexadas.
\end{abstract}

Aunque algunas investigaciones y proyectos guardan relación con el tema, fueron excluidos de este apartado aquellos cuyos paradigmas son diferentes a los utilizados en este estudio.

Se considera que la mayoría de las investigaciones tratan de presentar una situación en un momento determinado, por lo que los tamaños de las muestras no parecen suficientemente representativos para ser extrapolados a grupos de mayor tamaño. Sin embargo, dado el origen académico y científico de los artículos en estudio, se considera que la metodología de los mismos es válida.

En cuanto a la aplicación del metanálisis dentro de esta revisión sistemática, se considera inadecuado, pues las variables de los documentos analizados no son comparables entre sí por la heterogeneidad del tema.

\section{RESULTADOS}

Desde un enfoque legal, sociológico, educativo, médico, entre otros, en Costa Rica se han realizado diversas investigaciones respecto a los temas involucrados en este estudio. Como se verá más adelante, ya se han establecido relaciones entre discapacidad, nacimiento prematuro y estimulación temprana.

Sin embargo, en nuestro país, no se han desarrollado propuestas que vinculen ninguno de estos aspectos con las guarderías o Centros de Infantiles de Nutrición y Atención Integral (CEN-CINAI), institución del Ministerio de Salud de Costa Rica dedicada al cuido y atención de los infantes, hasta los seis años de edad, pertenecientes a las familias con menores ingresos económicos $y$, por ende, menores posibilidades de atención. Igual sucede con las Estancias del ISSSTEP (Instituto de Seguridad y Servicios Sociales de los Trabajadores al Servicio de los Poderes del Estado de Puebla) de la ciudad de Puebla en México, cuyas entidades atienden y ofrecen servicios de cuido a los hijos y las hijas de empleados públicos con edades desde los dos meses de edad hasta los seis años de vida.

A continuación, se mencionan las investigaciones relacionadas con los temas abarcados por este estudio, realizadas en Costa Rica y que, por sus enfoques, sirven de marco de referencia al resumir los avances científicos y académicos en estas áreas.

En cuanto al tema de la atención de la discapacidad, se han generado investigaciones y documentos como los siguientes:

En 1999, Marcela Rodríguez, Grethel Rodríguez, Sandra Rodríguez, Andreína Solís, Limberg Vargas y Edith Vega; proponen un Taller de actualización en discapacidad del recién nacido para profesionales en enfermería obstétrica de los Hospitales Calderón Guardia y México, como seminario de graduación para obtener la licenciatura de la Universidad de Costa Rica en enfermería con énfasis en salud de la mujer y perinatología. En el taller 
se trataron conceptos como discapacidad, minusvalía, invalidez, anomalía, deficiencia y discapacidad en el recién nacido, así como las principales malformaciones en Costa Rica, su incidencia y efectos.

Su objetivo era actualizar conocimientos y mejorar la atención de enfermería de niños, niñas y padres en forma interdisciplinaria, reforzando la intervención en crisis de primer orden que se debe brindar a la madre y a la familia, al darse el nacimiento de un recién nacido con alguna malformación evidente.

Entre las conclusiones a las que se llegó a través del taller están las siguientes:

»Existía, en ese momento, un mal manejo de los principales conceptos relacionados con la temática de discapacidad.

» Faltaba conocimiento respecto a datos sobre la incidencia de malformaciones congénitas en Costa Rica, los aspectos teóricos más relevantes y el manejo de dichas alteraciones.

»El profesional de enfermería no estaba asumiendo su papel protagónico en la valoración del recién nacido y su responsabilidad como informante en el diagnóstico.

»En los hospitales donde se realizó el taller no existían recursos o programas de apoyo para las familias de niños y niñas con discapacidad.

» Los padres con niños y niñas con alguna discapacidad esperan apoyo, información y orientación por parte del profesional en enfermería, ya que es con ellos que se tiene el contacto directo desde el nacimiento.

En el mismo año, Ana Grace Woodley Lewis, (1999) en su tesis para optar por la maestría en salud pública, La atención a la discapacidad dentro del marco de la reforma del sector salud: una propuesta de atención integral para la Región Atlántica, analizó la atención a la discapacidad existente en la región Atlántica de Costa Rica y propuso lineamientos para la atención integral.

La población en estudio fue todas las instituciones gubernamentales y no gubernamentales del Sector Salud, existentes en la Región Atlántica, que brindan servicios de atención a las personas con discapacidad.

Entre sus conclusiones se destacan:

» En ese momento, la atención a la discapacidad era deficiente, no se brindaba una atención integral en las instituciones del sector público, con excepción del Consejo Nacional de Rehabilitación y Educación Especial y el Centro Nacional de Rehabilitación.

»La atención a la discapacidad requería mayor compromiso y apoyo estatal.

»Las instituciones encargadas contaban con muy pocos recursos técnicos y tecnológicos para la detección y atención oportuna de la discapacidad.

El tema de la Estimulación Temprana Intrahospitalaria ha sido tratado, sobre todo, en propuestas para poner en marcha este tipo de servicio en diferentes hospitales del país. Entre las investigaciones más importantes encontramos:

En el 2006, Alicia González Gómez, Morrine Maitland Hilton y Angie Vargas Bonilla presentan a la Universidad de Costa Rica su tesis de maestría en Enfermería Pediátrica con énfasis en Neonatología: Consulta de enfermería pediátrica en estimulación temprana para los niños y niñas prematuros, de alto riesgo y con Síndrome de Down, egresados del Servicio de Neonatología del Hospital San Juan de Dios.

Su objetivo era establecer este tipo de consulta para la población de niños y niñas prematuros, de alto riesgo (asfixia perinatal) y alteraciones congénitas (Síndrome de Down), egresados del 
Servicio de Neonatología del Hospital San Juan de Dios en el 2005. Teniendo esta población características en común que afectan el desarrollo psicomotor y del lenguaje, se trabajó con 14 familias y su mayor logro fue la demostración de que tal servicio era factible y viable de operacionalizarse sin requerir recursos adicionales a los existentes en la institución. La formulación y ejecución del proyecto propuesto en esta tesis hizo posible su apertura en octubre del 2006.

En el mismo año, Celia Campos, Silvia Cordero, Gabriela Rojas y Mariela Vargas, en su práctica dirigida para obtener la licenciatura de enfermería en la Universidad de Costa Rica, crean un Programa de capacitación dirigido al personal de Enfermería, sobre Ludoterapia y estimulación temprana en la atención al niño y la niña hospitalizado(a) y su familia en los servicios de pediatría, neonatología y maternidad del Hospital San Rafael de Alajuela.

Su objetivo fue promover estrategias de intervención de enfermería basadas en Ludoterapia y estimulación temprana. La población con la que trabajaron fue el personal de enfermería asignado a laborar en los departamentos de pediatría, neonatología y maternidad de dicho Hospital (12 personas). Durante la práctica, obtuvieron los siguientes hallazgos:

»En la etapa de diagnóstico, existía conocimiento deficiente por parte de los profesionales de enfermería consultados en relación con la ludoterapia. Sin embargo, reconocían la importancia del juego para disminuir el impacto de la hospitalización, al minimizar la ansiedad de los niños y sus familias.

»Existían limitaciones de tiempo y cantidad de personal que obstaculizaban la aplicación de estas técnicas.

» La implementación del programa permitió descubrir que la terapia del juego rompía la rutina de los servicios hospitalarios y generaba emotividad y alegría, lo que ayudaba a los y las profesionales en enfermería a disfrutar más de su trabajo y ayudaba a canalizar la ansiedad y el estrés de los niños y de sus familias.

» Existía factibilidad para la incorporación de la ludoterapia en el plan de atención de enfermería, siempre y cuando se dieran capacitaciones al personal, además de disponibilidad de recursos y motivación.

En 2007, Marlene Jiménez Mora propone la Apertura de la consulta de enfermería pediátrica en estimulación temprana, para niños y niñas de alto riesgo o riesgo establecido de 0 a 6 años, en el Hospital San Rafael de Alajuela, en su tesis para optar por una maestría en enfermería pediátrica con énfasis en niñez y adolescencia, de la Universidad de Costa Rica.

A través de un diagnóstico epidemiológico y el desarrollo de un programa de atención temprana se logró el objetivo de institucionalizar dicho servicio. En el momento que se evalúo la consulta, los padres de los niños se mostraron satisfechos.

En cuanto a la cuestión de los nacimientos prematuros y los servicios que se pueden brindar a los niños y niñas en esta condición, se han realizado algunas propuestas para capacitar a los padres, por parte del personal de enfermería para brindar una atención especial a sus bebés que les permita una adecuada calidad de vida al regresar a su hogar. También se han llevado a cabo evaluaciones de la calidad de los servicios que los pequeños reciben en el centro hospitalario.

En la práctica dirigida, para obtener la licenciatura en salud de la mujer y perinatología de la Universidad de Costa Rica, realizada en 1992 por Sisinia Chacón y Silvia Tapia, se presentó un Programa de intervención de enfermería para padres de niños pretérmino del Instituto Materno Infantil Adolfo Carit.

El objetivo propuesto fue que, a través de dicho programa, se lograra una mejor adaptación de 
los niños y niñas prematuros al medio ambiente para mejorar su calidad de vida.

La muestra utilizada en este caso fueron 12 madres de niños y niñas pre término internados en aquel momento en el servicio de Neonatología del Instituto Carit (hoy Hospital de la Mujer).

A través de una evaluación de conocimientos y habilidades de las madres en cuanto a la atención de los niños pre término, en aspectos como: 1) Cuidados del recién nacido pre término; 2) Apego materno-paterno; 3) Estimulación temprana; 4) Lactancia materna, y un posterior desarrollo de un programa de atención para capacitar a dichas madres, se llegó a resultados como:

» Durante el pretest, el conocimiento en las áreas de cuidado del recién nacido pre término y lactancia materna tuvieron un nivel de acierto bajo, mientras las de apego materno y estimulación temprana tuvieron porcentajes de acierto más alto. Luego de la aplicación del programa, los porcentajes de conocimiento aumentaron.

» El nivel de "calidad de vida" de los niños y niñas prematuros mejoró mediante el conocimiento, la orientación y la motivación que adquirieron las madres durante el programa.

Para el 2005, con el tema: Programa de capacitación a madres y padres de neonatos pre término hospitalizados en el Servicio de Neonatología del Hospital Max Peralta de Cartago, Lidia Montero Jiménez presentó a la Universidad de Costa Rica su Práctica dirigida para optar por la licenciatura en enfermería.

Su objetivo al implementar dicho programa fue la capacitación de los padres y madres de niños y niñas pre término, para prepararlos para afrontar las necesidades especiales que presentarían los bebés al ser llevados a casa. La población con la que se trabajó fue 36 personas, padres y madres de bebés hospitalizados en el Servicio de Neonatología del Hospital Max Peralta.

Un resultado muy importante de este taller fue descubrir que el tiempo de estadía de los bebés en el hospital puede ser desde semanas hasta tres meses, con lo que se instó a aprovechar ese tiempo en la capacitación de los padres a través de diferentes medios, por ejemplo, material impreso y audiovisual.

En el 2006, Ana Patricia Alvarado, presentó la tesis Análisis de la calidad de la intervención del personal de enfermería en la atención integral del recién nacido (a) de pre término del Servicio de Neonatología del Hospital México para optar por la licenciatura en enfermería de la Universidad de Costa Rica.

Trabajando con un total de 10 personas que formaban parte del equipo de enfermería que trabajaba con los niños y niñas de pre término en el servicio de neonatología del hospital en estudio, se llega a las siguientes conclusiones:

» La falta de personal y la escasa motivación son aspectos que interferían en la calidad de la atención que se brinda en este servicio.

» En ese momento, existía escasa oportunidad para la capacitación y actualización del personal, lo que impactaba negativamente la calidad de la intercesión desde su aspecto científico.

»La manera en que se llevaban a cabo los procedimientos y el uso de la tecnología disponible fueron adecuados y contribuyó a mejorar la calidad del servicio.

En el 2010, para obtener el título de licenciatura en terapia física de la Universidad de Costa Rica, Gabriela Arias, Adriana Rodríguez y Jennifer Vega presentan el Diseño de una propuesta de atención fisioterapéutica para la intervención en la consulta externa de los niños y niñas prematuros, egresados de la Unidad de Neonatología del Hospital Rafael Ángel Calderón Guardia. 
Entre sus objetivos estaba proponer estrategias de atención fisioterapéutica para la estimulación psicomotriz de los niños y niñas prematuros en el hospital en cuestión. Con una población que incluía a todos los niños y niñas prematuros egresados de la Unidad de Neonatología del Hospital Calderón Guardia, durante el primer semestre del 2009, un total de 17 niñas y 25 niños, se presentan los siguientes hallazgos:

》

De los niños y niñas con mayor número de reflejos patológicos (de 7 a 11), la mayoría también presentó un número elevado de factores de riesgo (más de 5), sobretodo problemas respiratorios e ictericia.

Resulta evidente el interés de los padres porque sus hijos e hijas reciban esta clase de atención. Sin embargo, el hospital solo presta este servicio en casos muy serios.

Como se dijo anteriormente, no se han planteado investigaciones que relacionen los temas anteriores con los CEN-CINAI, pues, en cuanto a estos centros, se ha dado prioridad a la evaluación y desarrollo de propuestas relacionadas con: Salud (odontología, nutrición y prevención); personal (clima organizacional y capacitación); factores socioeconómicos de las poblaciones atendidas; gestión educativa y deserción.

En el caso de la República Mexicana, en relación con la estimulación temprana tenemos que:

En su artículo Frecuencia de desviaciones del neurodesarrollo a los 18 meses de edad en pacientes con alto riesgo neurológico que acuden a estimulación temprana, María de Lourdes Salinas Álvarez y Laura Peñaloza Ochoa resumen los resultados de un estudio retrospectivo, transversal, de una muestra de 87 pacientes ingresados al Programa de Estimulación Temprana Institucional del Hospital Infantil de México Federico Gómez, en sus primeros meses de vida, de enero de 2000 a octubre de 2006 . El 100\% mostró factores de riesgo biológico y alto riesgo neurológico.

De su investigación, las autoras infirieron que los Programas de Estimulación Temprana permiten una mejoría en la reorganización y madurez del sistema nervioso central, lo que permite la adquisición de habilidades y destrezas acordes con la edad. Aunque un $20.7 \%$ evolucionó con desviaciones del neurodesarrollo, solo el $5.7 \%$ presentó secuelas de graves a moderadas. Los tipos más comunes fueron parálisis cerebral infantil y el retardo en el desarrollo psicomotor global.

Entre otros hallazgos, las autoras concluyen que:

» Se debe realizar de forma temprana el diagnóstico de alto riesgo neurológico y complementarlo con seguimientos periódicos como pruebas neurológicas y coeficiente de desarrollo de Gesell, para mejorar la calidad de atención.

» Los factores de riesgo biológico y ambiental tienen un valor predictivo de las desviaciones del neurodesarrollo, por lo que deben ser registrados.

» Los Programas de Estimulación Temprana favorecen el desarrollo del sistema nervioso central, previniendo, mejorando o recuperando los daños estructurales y funcionales.

En diciembre del 2004, la Revista Mexicana de Pediatría publicó el artículo Estimulación temprana en niños menores de 4 años de familias marginadas, donde Manuel Pando Moreno junto a otros investigadores resume su estudio longitudinal de 7763 menores de 4 años distribuidos en 4472 de un grupo experimental y 3491 de un grupo control, estudiados por 40 semanas, en que fueron sometidos a estimulación temprana para valorar el efecto de dicha estimulación en niños cuando ésta es proporcionada por sus padres previamente capacitados. 
Entre los resultados obtenidos, se observó que el grupo experimental, antes de iniciar el programa de estimulación, tenía un promedio de 79.2 en el puntaje de desarrollo global mientras que en los niños del grupo control fue de 76.9; la diferencia fue 2.3. En cambio, después de la estimulación, la divergencia aumentó a 6.2, a favor del grupo experimental: 84.9 versus 78.7 . Aunque ambos grupos aumentaron su promedio, fue evidente el avance en el grupo experimental por 5.7 puntos más en su promedio de desarrollo.

Se ha abordado también el tema de la Neurohabilitación, por ejemplo, Eneida Porras Kattz y Thalía Harmony, en el 2007, en su artículo Neurohabilitación: un método diagnóstico y terapéutico para prevenir secuelas por lesión cerebral en el recién nacido y el lactante, describen esta metodología y su utilidad, reconociéndola como un proceso para la prevención de las secuelas de la lesión cerebral en recién nacidos, gracias a la plasticidad del sistema nervioso joven.

En el tema de prematuridad, Lorenzo Osorno Covarrubias, entre otros, en el artículo Factores maternos relacionados con prematuridad, presentado a la revista Ginecología y Obstetricia de México en el 2008 , realizan un estudio a través de una cohorte de recién nacidos vivos al nacer, con $500 \mathrm{~g}$ de peso o más, del 1 de enero de 2000 al 31 de diciembre de 2004, en el Centro Médico Ignacio García Téllez.

De 25355 nacimientos registrados, 11.9\% fueron prematuros (menos de 37 semanas de embarazo). Después del análisis de los porcentajes y las causas del nacimiento prematuro o cesárea, se obtuvieron las siguientes conclusiones:

» La ruptura prematura de membranas y la morbilidad materna durante el embarazo son las causas más comunes de prematuridad en dicho centro médico.

» Sin embargo, factores como: antecedentes maternos socioeconómicos y demográficos, de embarazos previos, de control y evolución del embarazo y de partos desfavorables también se relacionan con los nacimientos prematuros.

Más recientemente, en el año 2010, Guillermina Sánchez Rodríguez, entre otros, presentaron a la Revista Medicina Universitaria, el artículo Disminución del estrés del prematuro para promover su neurodesarrollo: nuevo enfoque terapéutico.

En este se explican las ventajas del NIDCAP (Newborn Individualized Developmental Care and Assessment Program), un programa de intervención basado en la observación del niño o la niña antes, durante y después de los procedimientos de cuidado por parte de personal calificado, con el fin de obtener resultados neuroconductuales positivos a largo plazo.

Las observaciones se concentran en los esfuerzos del neonato en alcanzar la autorregulación. Si se da una estimulación inapropiada el niño o la niña la rechaza o se estresa.

El programa es una forma de atención individualizada, que requiere la sensibilización y capacitación del personal médico. Sin embargo, se ha demostrado que estos programas enfocados en el desarrollo del recién nacido prematuro tienen buenos resultados tanto en el tiempo que el recién nacido se encuentra en el hospital como en su neurodesarrollo a largo plazo.

\section{RESULTADOS Y DISCUSION}

Como se mencionó anteriormente, no es posible aplicar métodos estadísticos convencionales a una serie de estudios con variables y poblaciones tan distintas, razón por la cual se procederá, tan sólo, a rescatar los aportes de estas investigaciones que son de relevancia para el objetivo de la investigación: Construir un protocolo de atención integral que incluya la estimulación y la atención temprana de las niñas y los niños nacidos prematuramente que presentan una condición de riesgo biológico. 
Habrá notado el lector que, en Costa Rica, las investigaciones se centraron, en el caso de la discapacidad, en la evaluación de la atención que se brindaba, tanto a nivel intrahospitalario como fuera de él, a las personas con discapacidad. En esos momentos (final de la década de los noventa), los resultados de las investigaciones demostraron deficiencias en la atención integral de las personas con discapacidad y sus familias. Sin embargo, en 1992, se presentó en el Instituto Materno Infantil Adolfo Carit (hoy Hospital de la Mujer) un programa para la capacitación de niños pre término.

Fue a partir del 2005 cuando, en Costa Rica, sobretodo, las y los futuros graduados en licenciaturas de enfermería, se preocuparon por proponer a las autoridades de diferentes hospitales la apertura y puesta en marcha de servicios institucionales de estimulación temprana y atención integral para niños prematuros, de alto riesgo o con alteraciones congénitas.

Cada uno de los estudios y planes piloto demostró aspectos positivos de la estimulación temprana, por lo menos en la mayor parte de los miembros de las poblaciones en estudio.

Otro aspecto importante que revelaron las investigaciones costarricenses es la importancia de capacitar, motivar y dotar a los profesionales en enfermería de los recursos necesarios para realizar adecuadamente la función en el proceso.

En el caso de México, es importante destacar el interés de sus investigadores por determinar la relación entre la estimulación temprana, el neurodesarrollo y la neurohabilitación, potenciando y demostrando en cada uno de sus estudios la importancia de la estimulación tempana en niños prematuros y con alto riesgo neurológico.

Además, la investigación de Manuel Pando (2004), que integró la variable de marginalidad, determinó el aumento del promedio de desarrollo en niños en esta condición, a través de la estimulación temprana, por lo que no es necesaria la existencia de prematuridad o alto riesgo para que los niños y niñas se beneficien de este grupo de técnicas.

Este hallazgo pone en evidencia el impacto positivo que tendrá la estimulación temprana en la mayoría de las poblaciones infantiles y, por ende, la importancia de esta como estrategia de potencialización del desarrollo, o bien, de prevención en los grupos que presentan alguna característica particular, sea de origen biológico o socioeconómico, que los hace más vulnerables.

\section{CONCLUSIONES}

Del análisis anterior se desprende:

» La prevención de la discapacidad a través de la estimulación temprana es fundamental, pero de igual forma lo son la capacitación y recursos con que cuenten los profesionales que atenderán a los niños y niñas con discapacidad, por lo que es necesario que se estudien las mismas variables que, en los años noventa, demostraron las deficiencias en este tema en Costa Rica, y de mantenerse las mismas condiciones implementar las medidas necesarias para solucionarlas.

»Las investigaciones y la teoría apuntan a que, cuánto más pronto se inicie la estimulación temprana, mayor impacto tendrá en la vida de los infantes, por la plasticidad del sistema nervioso central en los primeros meses de vida. De ahí que resulte vital capacitar y orientar a los padres de familia y docentes para que puedan ofrecer este apoyo a los niños y las niñas prematuros que presentan una condición de riesgo biológico.

»Es también necesaria la evaluación de los servicios institucionales que se empezaron a crear en los hospitales costarricenses para la atención de los niños y niñas pre término, a través de estimu- 
lación temprana y atención integral, con el fin de seguir la pista de sus logros y procesos.

\section{BIBLIOGRAFÍA}

Alvarado Bolaños, A. (2006). Análisis de la calidad de la intervención del personal de enfermería en la atención integral del recién nacido (a) de pre término, Servicio de Neonatología del Hospital México en 2006. Tesis para optar por la licenciatura en enfermería. Escuela de Enfermería, Universidad de Costa Rica. San José, Costa Rica.

Arias Bogantes, G., Rodríguez Obando, A. \& Vega López, J. (2010). Diseño de una propuesta de atención fisioterapéutica para la intervención en la consulta externa de los niños y niñas prematuros, egresados de la Unidad de Neonatología del Hospital Rafael Ángel Calderón Guardia. Seminario de Graduación para optar el título de licenciatura en terapia física. Escuela de Tecnologías en Salud, Universidad de Costa Rica. San José, Costa Rica.

Campos Campos, C., Cordero Castro, S., Rojas Alfaro \& G. Vargas Bolaños, M. (2006). Programa de capacitación dirigido al personal de enfermería, sobre ludoterapia y la estimulación temprana en la atención del niño y la niña hospitalizado(a) y su familia: en los servicios de pediatría, neo- natología y maternidad, Hospital San Rafael de Alajuela, 2006. Práctica dirigida para optar por la licenciatura en enfermería. Escuela de Enfermería, Universidad de Costa Rica. San José, Costa Rica.

Chacón Ugalde, S. \& Tapia Cerdas, S. (1992). Programa de intervención de enfermería para padres de niños pre término, Instituto Materno Infantil Adolfo Carit. Práctica dirigida para optar por el título de licenciatura en salud de la mujer y perinatología. Escuela de Enfermería, Universidad de Costa Rica. San José, Costa Rica.

González Gómez A., Maitland Hilton, M. \& Vargas Bonilla, A. (2006). Consulta de enfermería pediátrica en estimulación temprana para los niños y niñas prematuros, de alto riesgo y con Síndrome Down, egresados del Servicio de Neonatología del Hospital San Juan de Dios. Tesis para optar por la maestría en pediátrica con énfasis en neonatología. Sistema de Estudios de Posgrado, Universidad de Costa Rica. San José, Costa Rica.

Jiménez Mora, M. (2007). Apertura de una consulta de enfermería pediátrica en estimulación temprana, para niños y niñas de alto riesgo o riesgo establecido de o a 6 años en el Hospital San Rafael de Alajuela. Tesis para optar por el título máster en enfermería pediátrica con énfasis en niñez y adolescencia. Sistema de Estudios de Posgrado, Universidad de Costa Rica. San José, Costa Rica.

Montero Jiménez, L. (2005). Programa de capacitación a madres y padres de neonatos pretérmino hospitalizados en el Servicio de Neonatología del Hospital Max Peralta de Cartago. Práctica dirigida para optar por el título de licenciatura en enfermería. Escuela de Enfermería, Universidad de Costa Rica. San José, Costa Rica.

Osorno Covarrubias, L., Rupay Aguirre, G. E., Rodríguez Chapuz, J., Lavadores May, A. I., Dávila Velázquez, J. y Echeverría Eguiluz, M. (2008). Factores maternos relacionados con prematuridad [Versión electrónica]. Ginecología y Obstetricia de México, 76(9), 526-536.

Porras Kattz, E. y Harmony, T. (2007) Neurohabilitación: un método diagnóstico y terapéutico para prevenir secuelas por lesión cerebral en el 
recién nacido y el lactante [Versión electrónica]. Boletín Médico del Hospital Infantil de México, 64, 125-135.

Pando Moreno, M., Aranda Beltrán, C., Amezcua Sandoval, M. T., Salazar Estrada, J. G. y, Torres López, T.M. (2004). Estimulación temprana en niños menores de 4 años de familias marginadas [Versión electrónica]. Revista Mexicana de Pediatría, 71(6), 273-277.

Rodríguez Rodríguez, G., Rodríguez Rojas, S., Solís González, A., Vargas Araya, L. \& Vega Calvo, E. (1999). Taller de actualización en discapacidad del recién nacido para profesionales en enfermería obstétrica de los Hospitales Calderón Guardia y México. Mayo 1999. Seminario de graduación para optar por el título de licenciatura en enfermería con énfasis en salud de la mujer y perinatología. Escuela de Enfermería, Universidad de Costa Rica. San José, Costa Rica.

Salinas Álvarez, M. y Peñaloza Ochoa, Laura. (2007). Frecuencia de desviaciones del neuro- desarrollo a los 18 meses de edad en pacientes con alto riesgo neurológico que acuden a estimulación temprana [Versión electrónica]. Boletín Médico del Hospital Infantil de México, 64, 214-220.

Sánchez Rodríguez, G., Quintero Villegas, L., Rodríguez Camelo, G., Nieto Sanjuanero, A. y Rodríguez Balderrama, I. (2010). Disminución del estrés del prematuro para promover su neurodesarrollo: nuevo enfoque terapéutico [Versión electrónica]. Medicina Universitaria, 12(48), 176-180.

Woodley Lewis, A. (1999). La atención a la discapacidad dentro del marco de la reforma del sector salud: una propuesta de atención integral para la Región. Tesis para optar por la maestría en salud pública con mención en gerencia de servicios de salud. Sistema de Estudios de Posgrado, Universidad de Costa Rica. San José, Costa Rica. 